\title{
Continuous Modulation of Action Potential Firing by a Unitary GABAergic Connection in the Globus Pallidus In Vitro
}

\author{
Jenia Bugaysen, ${ }^{1}$ Izhar Bar-Gad, ${ }^{2}$ and Alon Korngreen ${ }^{1,2}$ \\ ${ }^{1}$ The Mina and Everard Goodman Faculty of Life Sciences and ${ }^{2}$ The Leslie and Susan Gonda Interdisciplinary Brain Research Center, Bar-Ilan University, \\ Ramat Gan 52900, Israel
}

\begin{abstract}
The firing patterns of neurons in the globus pallidus (GP) are affected by two major sources of GABAergic inhibition: striatal afferents and local axon collaterals. Local GABAergic GP-GP synapses display short-term depression (STD) and very sparse connectivity. At the high presynaptic firing rates typical in the GP, one would expect this STD to be complete, practically canceling the postsynaptic impact of the synapse. To investigate the apparent paradox of a synapse not affecting its postsynaptic neuron, we performed dual whole-cell recordings in acute brain slices from rats and recorded, for the first time, unitary IPSPs from a GP-GP GABAergic connection. We show that at high presynaptic firing rates the unitary connection continuously modulates the postsynaptic firing rate through a combination of large chloride driving force, unitary IPSP summation, and incomplete synaptic depression. Our findings indicate that, despite substantial STD and sparse connectivity, local GABAergic axon collaterals in the GP may echo the changes in presynaptic firing frequency across postsynaptic targets.
\end{abstract}

\section{Introduction}

The globus pallidus (GP) integrates multiple streams of synaptic input in the basal ganglia (DeLong, 2000). GP function is mainly controlled by GABAergic input from the striatum (François et al., 1984; Kita and Kitai, 1994), GABAergic collaterals of neighboring GP neurons (Sato et al., 2000), and glutamatergic inputs from the subthalamic nucleus (STN) (Smith and Parent, 1988). Several studies have shown that short-term depression (STD) evoked by extracellular stimulation can affect firing dynamics in GP neurons in vitro by altering the timing of GP spiking (Rav-Acha et al., 2005; Sims et al., 2008; Miguelez et al., 2012). STD also shapes excitatory inputs reaching the GP from the STN (Hanson and Jaeger, 2002). Although this STD affects the firing of GP neurons, its role in network activity remains unclear.

GP collaterals form a sparse inhibitory network in the GP with a connectivity probability of $\sim 1 \%$ (Sadek et al., 2007). In the behaving rat GP neurons fire tens of action potentials (APs) per second (Benhamou et al., 2012), a firing frequency at which STD could potentially be complete. This sparse and synaptically depressed network could thus have a negligible effect on neuronal

\footnotetext{
Received May 10, 2013; revised June 11, 2013; accepted June 27, 2013.

Author contributions: J.B., I.B.-G., and A.K. designed research; J.B. and A.K. performed research; J.B., I.B.-G., and A.K. analyzed data; J.B., I.B.-G., and A.K. wrote the paper.

This work was supported by the Legacy Heritage Bio-Medical Program of the Israeli Science Foundation to A.K. and I.B.-G. (Grant \#981/10). We thank Adi Roth for endless hours on the Neurolucida and Yura Grishko for graphical help.

The authors declare no competing financial interests.

Correspondence should be addressed to Dr. Alon Korngreen, Bar-Ilan University, The Leslie and Susan Gonda Interdisciplinary Brain Research Center, The Mina and Everard Goodman Faculty of Life Sciences, Ramat Gan 52900, Israel. E-mail: alon.korngreen@biu.ac.il.

DOI:10.1523/JNEUROSCI.1970-13.2013

Copyright $\odot 2013$ the authors $\quad 0270-6474 / 13 / 3312805-05 \$ 15.00 / 0$
}

activity in the GP. If so, then what is its role? Could this network be redundant in the normal state?

To investigate this question we performed dual whole-cell recordings from pairs of synaptically connected GP neurons, enabling us to investigate the dynamic properties of this unitary synapse. We show that, despite sparseness and STD, unitary GABAergic synaptic connection in the GP can significantly affect postsynaptic firing patterns in vitro, thus possibly playing an important role in the information processing within this nucleus.

\section{Materials and Methods}

In vitro slice preparation and solutions. Sagittal slices (300 $\mu \mathrm{m}$ thick) of rat brain containing cortex, striatum, and GP were prepared as described previously (Bugaysen et al., 2010, 2011). Wistar rats (16-22 d old) of either sex were killed by rapid decapitation according to the guidelines of the Bar-Ilan University Animal Welfare Committee. This procedure was approved by the National Committee for Experiments on Laboratory Animals at the Israeli Ministry of Health. The brain was quickly removed and placed in ice-cold artificial CSF (ACSF) containing the following (in mM): $125 \mathrm{NaCl}, 4 \mathrm{KCl}, 25 \mathrm{NaHCO}_{3}, 1.25 \mathrm{Na}_{2} \mathrm{HPO}_{4}, 2 \mathrm{CaCl}_{2}, 2 \mathrm{MgCl}_{2}, 25$ glucose, and $0.5 \mathrm{Na}$-ascorbate, $\mathrm{pH} 7.4$, with $95 \% \mathrm{O}_{2} / 5 \% \mathrm{CO}_{2}$. Slices were cut with an HR2 Slicer (Sigman Electronic) and transferred to a submersion-type chamber, where they were maintained for the remainder of the day constantly perfused with oxygenated ACSF and held at $34^{\circ} \mathrm{C}$. In several experiments bicuculline methiodide was added to ACSF to block $\mathrm{GABA}_{\mathrm{A}}$ receptors (final concentration $50 \mu \mathrm{M}$ ). The GP nucleus and individual GP neurons were visualized using infrared differential interference contrast microscopy. The standard pipette solution contained the following (in $\mathrm{mm}$ ): $140 \mathrm{~K}$-gluconate, $10 \mathrm{NaCl}, 10$ HEPES, 4 MgATP, 0.05 Spermin, 5 L-glutathione, 0.2 EGTA, 0.4 GTP, and 0.2\% biocytin (Sigma), pH 7.2, with KOH. The reference electrode was an $\mathrm{Ag}-\mathrm{AgCl}$ pellet placed in the bath. The $10 \mathrm{mV}$ liquid junction potential measured under the ionic conditions reported here was not corrected for. 

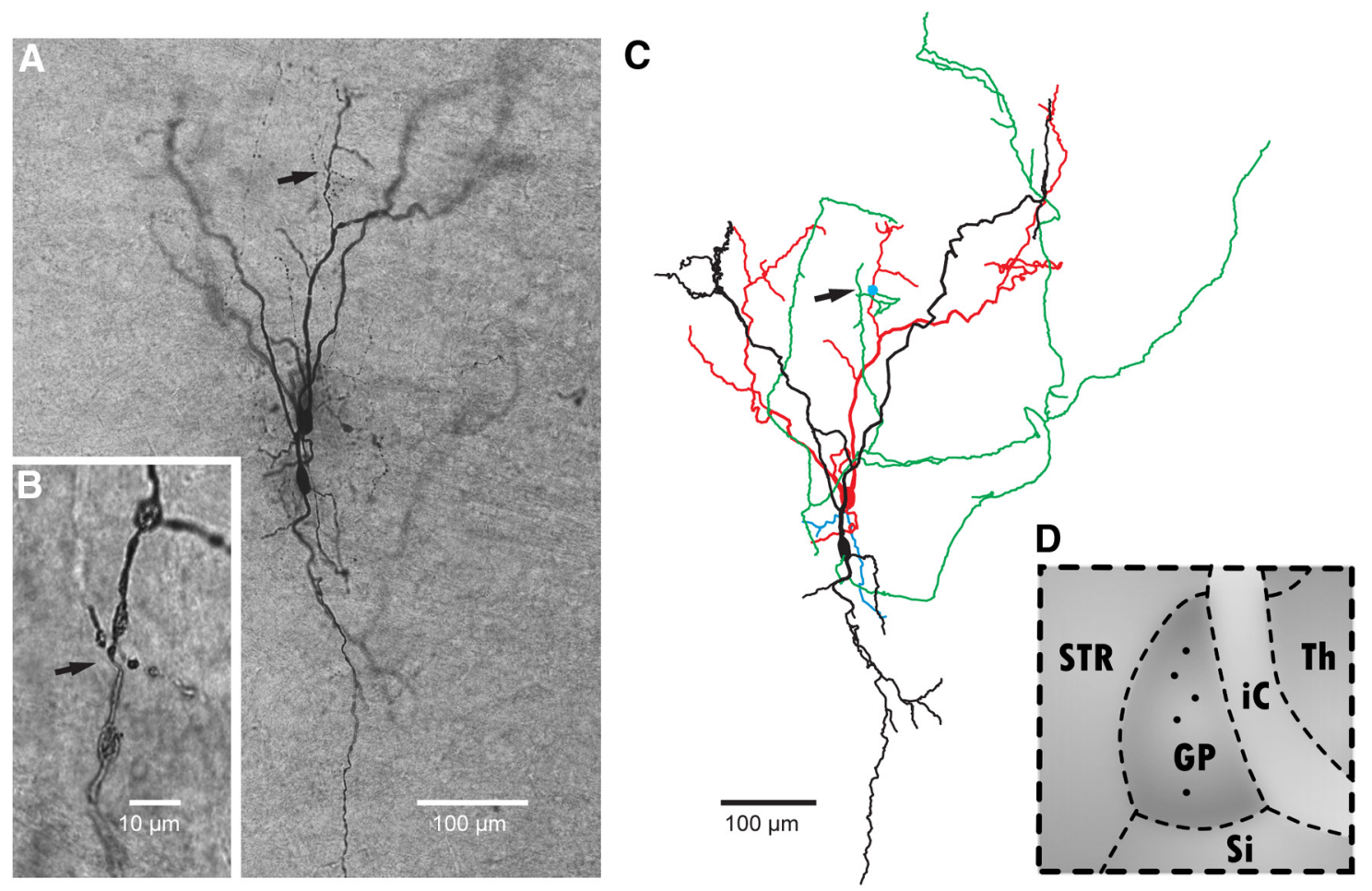

Figure 1. Morphological analysis of synaptically connected pairs of GP neurons. $A$, Digital image of two biocytin filled and stained GP neurons in a brain slice. Arrow marks the location of a putative synaptic connection. $\boldsymbol{B}$, Enlargement of the location of the putative synaptic connection. $\boldsymbol{C}$, A Neurolucida reconstruction of the pair of cells in $\boldsymbol{A}$. Black, presynaptic soma and dendrites; green, presynaptic axon; red, postsynaptic soma and dendrites; blue, postsynaptic axon. Arrow indicates location of the putative synaptic connection (emphasized by filled pale blue circle). $\boldsymbol{D}$, Schematic drawing of the GP and surrounding brain areas. The locations of the five paired recordings are marked by filled circles.

A

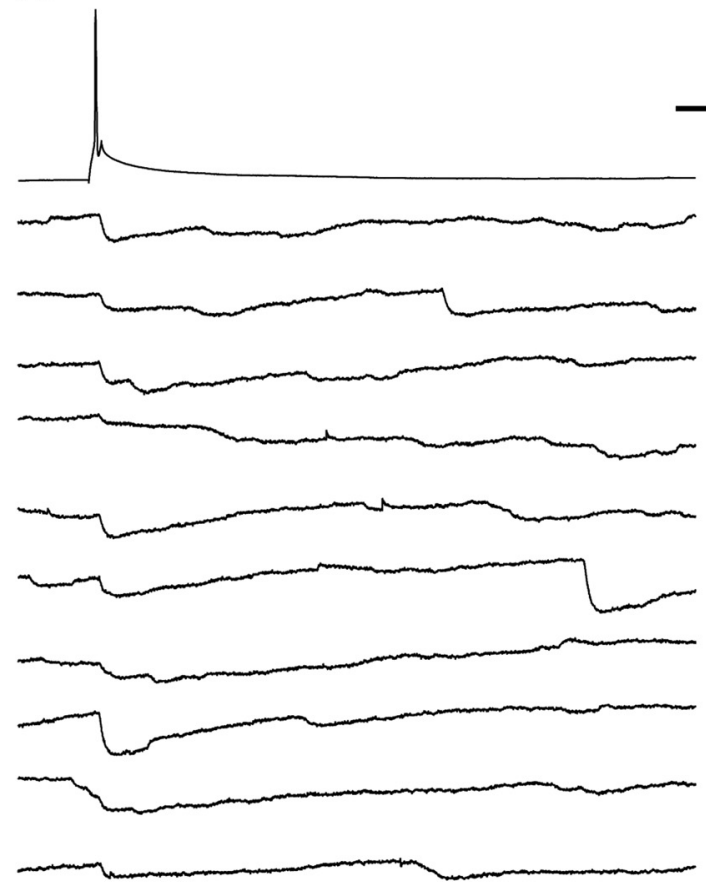

B

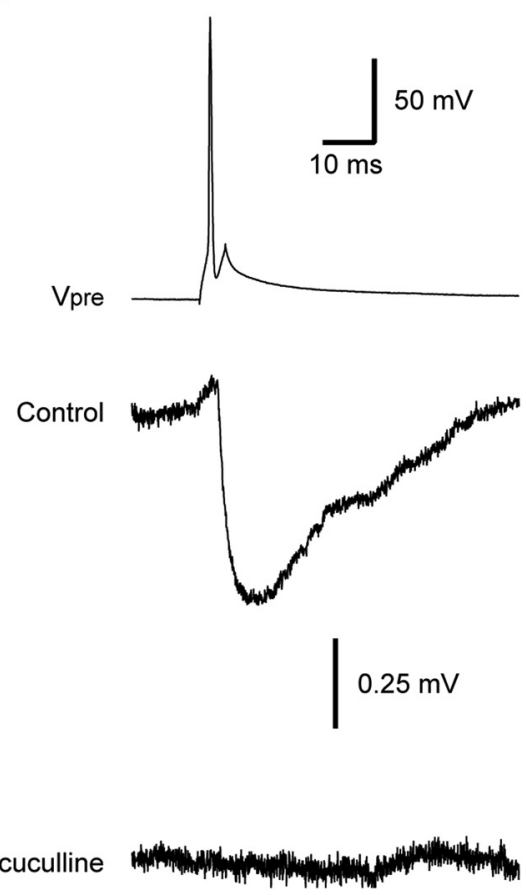

Figure 2. Presynaptic APs generate unitary IPSPs in GP neurons. $A$, Representative recording of a presynaptic AP aligned with 10 consecutive traces of the postsynaptic membrane potential. $\boldsymbol{B}$, Representative recording of a presynaptic AP aligned to an average of 40 consecutive traces of the postsynaptic membrane potential. Addition of $50 \mu \mathrm{m}$ bicuculline eliminated the IPSP (bottom trace, also an average of 40 membrane potential traces).

Electrophysiology and histology. Dual whole-cell recordings from somata of two GP neurons were made using a Multiclamp 700B amplifier (Molecular Devices). Voltage was filtered at $10 \mathrm{kHz}$ and sampled at 50 or $20 \mathrm{kHz}$ using Axoclamp 9 (Molecular Devices), digitized by a Digidata
1320 interface (Molecular Devices), and stored on the hard disk of a personal computer. Patch pipettes $(4-7 \mathrm{M} \Omega$ ) were pulled from thickwalled borosilicate glass capillaries $(2.0 \mathrm{~mm}$ outer diameter, $0.5 \mathrm{~mm}$ wall thickness; Hilgenberg). At the end of each experiment slices were fixed in 
cold 100 mm PBS, pH 7.4, containing 4\% paraformaldehyde. After fixation, slices were incubated for $2 \mathrm{~h}$ in avidin-biotinylated horseradish peroxidase (ABC-Elite; Vector Laboratories) and the stain developed using $0.015 \%$ diaminobenzidine. The stained neurons were digitally traced using a Neurolucida system (MicroBrightField). To verify the identification of putative synaptic contacts two observers independently reconstructed the same cell pairs. Putative synaptic contacts were selected only if both reconstructions were in agreement.

Data analysis. Off-line analyses were performed using Offline Sorter 2.8.6 (Plexon), NeuroExplorer 4.007 (Nex Technologies), MATLAB R2007b (MathWorks) and Igor Pro 6.0 (WaveMetrics) on a personal computer. Results are displayed as mean \pm SD unless otherwise stated.

\section{Results}

Anatomical studies suggest that the probability of connectivity of axon collaterals of GABAergic neurons in the GP is 1\% (Sadek et al., 2007). We observed a synaptic connection between 5 of the 173 pairs of GP neurons we recorded $\left(5 /\left(2^{\star} 173\right) \approx 1.4 \%\right.$ probability of connectivity). The somata of the connected pairs were separated by a mean distance of $84 \pm 85 \mu \mathrm{m}(n=5$, range $20-220$ $\mu \mathrm{m})$. Figure $1 A$ shows the image of a representative pair. Each pair was examined under light microscopy to identify putative connections between the axon and dendrite (Fig. 1B) and the pairs were reconstructed (Fig. $1 C$ ). This qualitative analysis identified putative synaptic connections in three of the five pairs. In each case one putative synaptic contact was observed on one of the postsynaptic dendrites at an average distance of $230 \mu \mathrm{m}$ from the soma (range 150-310 $\mu \mathrm{m}$ ). All five pairs recorded were at least $300 \mu \mathrm{m}$ from the boundary of the GP (Fig. 1D). It has been suggested that a single GP neuron medial to the GP boundary has $\sim 600$ synaptic terminals and forms several synaptic contacts with other GP neurons and that some of these contacts are proximal to the soma (Sadek et al., 2007). Due to some biocytin spill from the patch-clamp electrodes the vicinity of the somata could not be fully resolved leading to the conclusion that our morphological analysis may have underestimated the number of synaptic contacts between GP neurons. Thus, It was not possible to determine the full extent of the number of synaptic terminals made by each GP axon on postsynaptic targets within the GP.

All five connected pairs displayed unidirectional synaptic connections; no reciprocal synaptic connections were observed. Four pairs were sufficiently stable to perform quantitative electrophysiology. Presynaptic APs elicited robust ISPSs in the postsynaptic neuron (Fig. $2 A$ ). In the representative experiment shown in Figure $2 A$ both presynaptic and postsynaptic neurons were hyperpolarized by a constant current (typically -20 to $-30 \mathrm{pA}$ ) injected via the patch pipette to eliminate spontaneous AP firing. Figure $2 B$ shows the IPSP averaged from 40 repetitive sweeps. Average IPSP amplitude was $0.4 \pm 0.5 \mathrm{mV}(n=4)$ with a synaptic delay of $0.96 \pm 0.27 \mathrm{~ms}(n=4)$. Mono-exponential functions fitted to the rise and decay quantified the rise time constant $(5 \pm$ $3 \mathrm{~ms}, n=4)$ and the decay time constant ( $40 \pm 23 \mathrm{~ms}, n=4)$. As a last step in two experiments we applied the GABAergic antagonist bicuculline (50 $\mu \mathrm{M})$; this completely blocked the IPSP (Fig. 2).

The dynamic properties of the unitary synaptic connections could be quantitatively measured in two experiments. Both presynaptic and postsynaptic neurons were hyperpolarized with a small constant current injected via the patch pipette. Five action potentials were triggered in the presynaptic neurons at fixed intervals. Figure $3 A$ displays the average of 30 traces of the postsynaptic membrane potential following this stimulation protocol. Stimulating the presynaptic neuron with a $10 \mathrm{~Hz}$ train gave shallow STD between successive IPSPs. Substantial STD was observed when the train frequency was raised to $40 \mathrm{~Hz}$ (Fig. $3 A$ ).
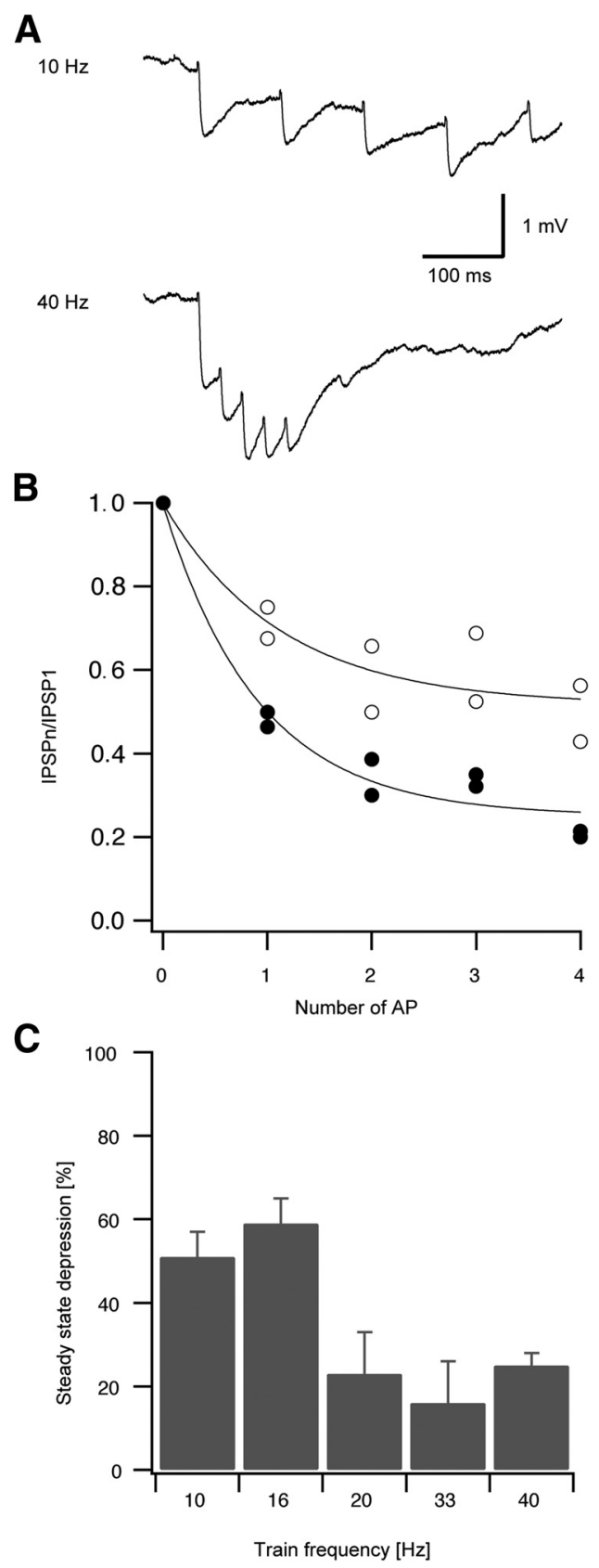

Figure 3. STD of unitary GP synapses. $\boldsymbol{A}$, Average of 30 consecutive traces of postsynaptic membrane potentials generated following presynaptic stimulation with a train of five APs at 10 $\mathrm{Hz}$ (top trace) or five APs at $40 \mathrm{~Hz}$ (bottom trace). $\boldsymbol{B}$, The ratio of the $n$th IPSP in the train to the first IPSP was plotted as a function of the number of the AP in the train. $\bigcirc$, two synapses stimulated at $10 \mathrm{~Hz} ; 0$, two synapses stimulated at $40 \mathrm{~Hz}$. Smooth lines are exponential curve fits to each dataset. C, Steady-state depression values extracted from curve fits similar to those in $\boldsymbol{B}$ plotted as a function of the frequency of the presynaptic $A P$ train. Error bars indicate the least-square error of the exponential curve fit in $\boldsymbol{B}$.

Since we were working only in current-clamp mode, the IPSPs summated, hyperpolarizing the membrane potential (Fig. $3 A$ ). To estimate the steady-state depression generated by each presynaptic stimulation frequency we fitted an exponential function to the normalized amplitudes of the IPSPs (Fig. $3 B$ ). This analysis suggested that at frequencies $>20 \mathrm{~Hz}$ the steady-state depression of the synapse was $\sim 80 \%$ (Fig. $3 C$ ).

The summation of the IPSPs at high presynaptic stimulation frequencies and the incomplete STD at these frequencies suggests 
the following possible biophysical mechanism for the function of this synapse. Depressed IPSPs summate during a highfrequency train of presynaptic APs. Since the membrane potential of a GP neuron is typically close to AP threshold, the chloride driving force is relatively large. Therefore, unitary IPSP summation could hyperpolarize the membrane potential pulling it away from AP threshold (Fig. 3A).

To test this mechanism we hyperpolarized the presynaptic neuron by a constant current injection of $-25 \mathrm{pA}$ but allowed the postsynaptic neuron to fire spontaneously. We then stimulated the presynaptic neuron with a $40 \mathrm{~Hz}$ train for $40 \mathrm{~s}$ (Fig. $4 A$ ). As predicted, an immediate and prolonged reduction of the postsynaptic firing frequency was observed (Fig. 4A). The spike-triggered average of the postsynaptic membrane potential demonstrated a clear average IPSP (Fig. 4B). This average IPSP was similar to that calculated using only the second half of the stimulation train (Fig. 4C). That is, as suggested above, STD was not complete, allowing the IPSPs to summate and affect postsynaptic firing.

At onset of the $40 \mathrm{~Hz}$ stimulation the mean postsynaptic firing rate decreased by $52 \pm 12 \%$, stabilizing to a $27 \pm 14 \%$ decrease during the last $40 \mathrm{~s}$ of stimulation $(n=3$; Fig. $4 D$ ). The overall decrease in the firing rate derived from a transient decrease in firing rate following each synaptic event (Fig. 4E). This decrease was dramatic even during clear STD, reaching a $64 \pm 13 \%$ decrease with an overall period of significant decrease of 4-13 ms following the presynaptic activity (Fig. $4 E$ ). The rate then returned to baseline for the remainder of the interstimulus interval. That is, the overall firing rate comprised a period of severe decrease that averaged with a period showing no decrease.

\section{Discussion}

We present the first recordings of unitary GABAergic postsynaptic potentials in GP neurons. Our results corroborate previous anatomical studies suggesting low connectivity between two GP neurons (here $\sim 1.4 \%$ ). Extracting some STD properties of the synapses (Fig. 3), we demonstrated that a unitary GABAergic synaptic connection could modulate the postsynaptic firing rate during prolonged high-frequency presynaptic stimulation (Fig. 4).

A simple mechanism may allow a unitary GABAergic connection to significantly affect postsynaptic firing. First, since the postsynaptic neurons fire spontaneously they are far from the chloride reversal potential. Thus, the large driving force will boost even a small inhibitory synaptic conductance. Second, although the synapse is depressed by $\sim 80 \%$ at high presynaptic firing, some GABA will still be released. Third, due to the slow decay of the IPSP, the activation of the synapse at high frequency, typical to these GP neurons, leads to postsynaptic GABAergic
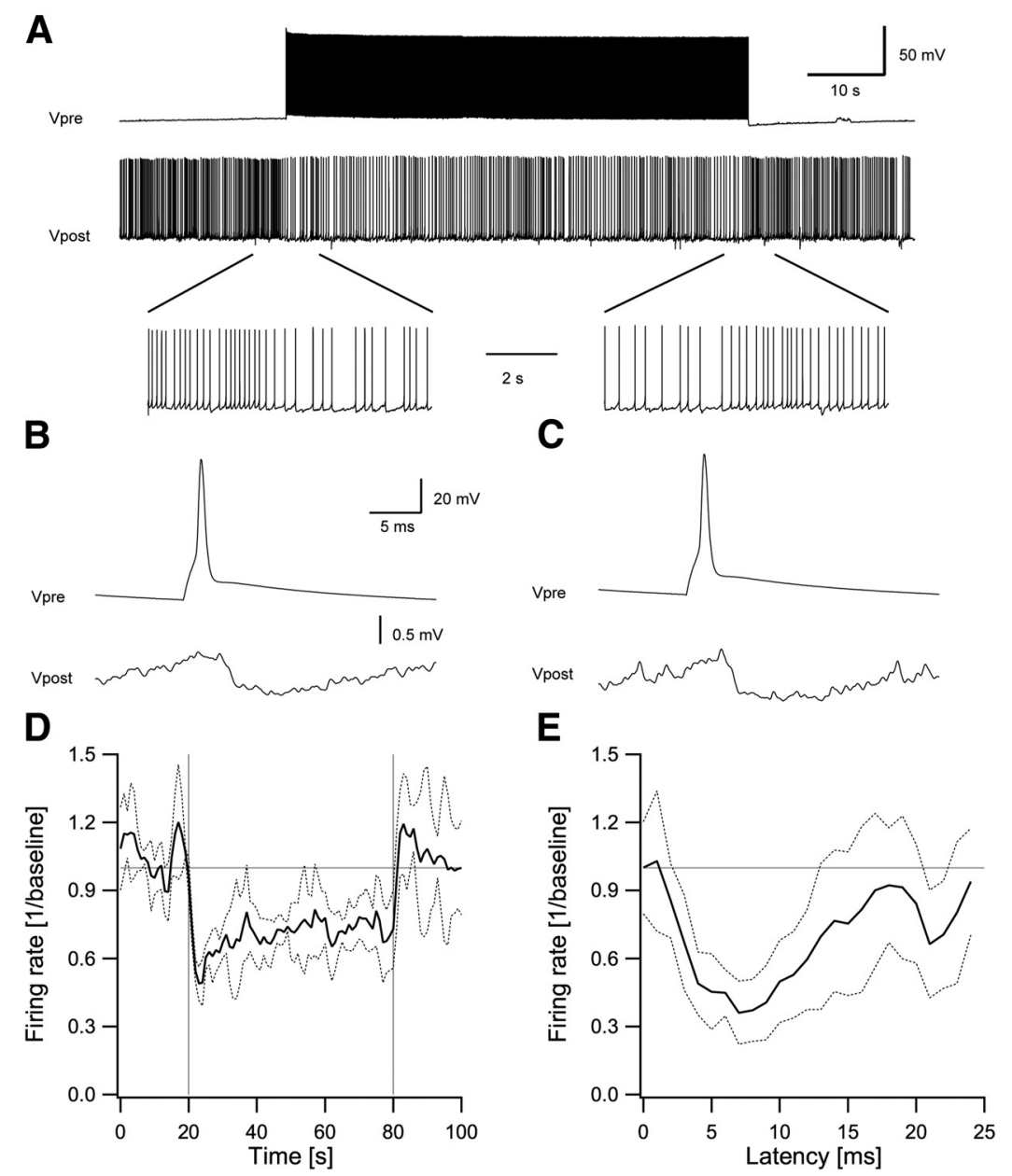

Figure 4. A unitary inhibitory synaptic connection can modulate postsynaptic firing of a GP neuron. $\boldsymbol{A}$, Representative plot of presynaptic and postsynaptic membrane potential when a $40 \mathrm{~s}$ train of $40 \mathrm{~Hz}$ was generated in the presynaptic neuron. The me histogram of the postsynaptic neuron $(n=3)$ aligned to the stimulation pulses and normalized to the prestimulation mean firing rate. Dashed lines mark \pm 1 STD of firing frequency before stimulus onset.

synaptic integration (Fig. 3A). This summation, combined with the large chloride driving force, hyperpolarizes the neuron, resulting in prolonged reduction of the postsynaptic firing frequency (Fig. 4). Thus, coupling a depressing synapse with highfrequency presynaptic firing effectively transforms the discrete action of the synapse into a continuous one. It is important to note that the overall firing rate modulation comprises of a period of a large decrease that averages with a period showing no decrease (Fig. 4E). Thus, a higher presynaptic firing rate will affect continuously postsynaptic activity by modulating the ratio of the decreased versus nondecreased firing.

An important component of this suggested mechanism is the slow IPSP decay kinetics. Interestingly, the IPSP decay kinetics reported here is slower than those reported by a previous study recording IPSCs in GP neurons following extracellular stimulation (Sims et al., 2008). Our recordings were made in the currentclamp mode and were influenced by the membrane time constant as opposed to previous voltage-clamp recordings (Sims et al., 2008). Moreover, It has been shown that at depolarized mem- 
brane potentials, close to AP threshold, the hyperpolarization induced by the IPSP inactivates voltage-gated sodium channels greatly prolonging IPSP decay (Stuart, 1999). This rectification has been shown to increase the ability of IPSPs to modulate AP firing (Stuart, 1999). This possible mechanism for increasing IPSP decay time may change integration of GP neurons around AP threshold.

In addition to the local axon collateral investigated in this study, GP neurons project to other basal ganglia nuclei. It has been recently shown in vitro, using extracellular stimulation, that the synapses of GP axons onto neurons in the STN display similar dynamics to those observed by us (Atherton et al., 2013). The major conclusion of that study is that, despite substantial depression, the GP-STN GABAergic synapse can effectively modify the firing of STN neurons (Atherton et al., 2013). While it is probable that GP-GP and GP-STN synapse may differ in multiple ways, this study provides important support to a view of the GP and STN interaction leaning toward continuous impact of synaptic input.

Caution must be used when trying to extend our findings into the in vivo domain and attempting to predict the function of the GP network. The extent of change in the firing rate of a GP neuron in vivo by a single GABAergic synapse is likely to be smaller since the impact of the unitary inhibition is enhanced in the slice preparation due to the reduced excitatory drive and higher input resistance of the cell. However, nonlinear mechanisms, such as the possible IPSP boosting discussed above, may offset for these slice distortions in the in vivo condition allowing a single synapse to have a large distinct effect. Clearly, a quantitative mapping of the nonlinear properties of GP neurons onto the firing properties of these neurons will improve our ability to better understand synaptic integration of these neurons.

In vivo most cells in the GP fire at high frequencies (Elias et al., 2007; Benhamou et al., 2012). Our results suggest that during high-frequency firing of the presynaptic neuron the firing probability of its postsynaptic neuron decreases. Excitatory input from the STN to a single GP neuron may decrease the firing probability of the neurons postsynaptic to this GP neuron, while inhibitory input from the striatum may have the opposite effect. This modulation of firing probability can affect both frequency and pattern of firing within the GP. While highly speculative, this may increase variability in the firing activity of GP neurons. The partially closed corticobasal ganglia loop inherently reduces correlations in the normal state (Bar-Gad et al., 2003). Prior hypotheses have focused on the roles of the striatum in this process, using either collateral inhibition between spiny projection neurons (Tunstall et al., 2002) or GABAergic interneurons (Koós and Tepper, 1999). One possible hypothesis stemming from our results is that GP-GP collaterals may also partake in this information processing. Most studies of the basal ganglia and its related disorders regard the GP as an internal relay station. Establishing the role of the local GP network is important in changing this concept. Understanding the role of this local network in the normal state and the changes it undergoes during various pathologies may provide a novel target for intervention in basal ganglia disorders.

\section{References}

Atherton JF, Menard A, Urbain N, Bevan MD (2013) Short-term depression of external globus pallidus-subthalamic nucleus synaptic transmission and implications for patterning subthalamic activity. J Neurosci 33:71307144. CrossRef Medline

Bar-Gad I, Morris G, Bergman H (2003) Information processing, dimensionality reduction and reinforcement learning in the basal ganglia. Prog Neurobiol 71:439-473. CrossRef Medline

Benhamou L, Bronfeld M, Bar-Gad I, Cohen D (2012) Globus pallidus external segment neuron classification in freely moving rats: a comparison to primates. PLoS One 7:e45421. CrossRef Medline

Bugaysen J, Bronfeld M, Tischler H, Bar-Gad I, Korngreen A (2010) Electrophysiological characteristics of globus pallidus neurons. PLoS One 5:e12001. CrossRef Medline

Bugaysen J, Bar-Gad I, Korngreen A (2011) The impact of stimulation induced short-term synaptic plasticity on firing patterns in the globus pallidus of the rat. Front Syst Neurosci 5:16. Medline

DeLong MR (2000) The basal ganglia. In: Principles of neural science, Ed 4 (Kandel ER, Schwartz JH, Jessell TM, eds), pp 853-867. New York: McGraw Hill.

Elias S, Joshua M, Goldberg JA, Heimer G, Arkadir D, Morris G, Bergman H (2007) Statistical properties of pauses of the high-frequency discharge neurons in the external segment of the globus pallidus. J Neurosci 27: 2525-2538. CrossRef Medline

François C, Percheron G, Yelnik J, Heyner S (1984) A Golgi analysis of the primate globus pallidus. I. Inconstant processes of large neurons, other neuronal types, and afferent axons. J Comp Neurol 227:182-199. CrossRef Medline

Hanson JE, Jaeger D (2002) Short-term plasticity shapes the response to simulated normal and parkinsonian input patterns in the globus pallidus. J Neurosci 22:5164-5172. Medline

Kita H, Kitai ST (1994) The morphology of globus pallidus projection neurons in the rat: an intracellular staining study. Brain Res 636:308-319. CrossRef Medline

Koós T, Tepper JM (1999) Inhibitory control of neostriatal projection neurons by GABAergic interneurons. Nat Neurosci 2:467-472. CrossRef Medline

Miguelez C, Morin S, Martinez A, Goillandeau M, Bezard E, Bioulac B, Baufreton J (2012) Altered pallido-pallidal synaptic transmission leads to aberrant firing of globus pallidus neurons in a rat model of Parkinson's disease. J Physiol 590:5861-5875. CrossRef Medline

Rav-Acha M, Sagiv N, Segev I, Bergman H, Yarom Y (2005) Dynamic and spatial features of the inhibitory pallidal GABAergic synapses. Neuroscience 135:791-802. CrossRef Medline

Sadek AR, Magill PJ, Bolam JP (2007) A single-cell analysis of intrinsic connectivity in the rat globus pallidus. J Neurosci 27:6352-6362. CrossRef Medline

Sato F, Lavallée P, Lévesque M, Parent A (2000) Single-axon tracing study of neurons of the external segment of the globus pallidus in primate. J Comp Neurol 417:17-31. CrossRef Medline

Sims RE, Woodhall GL, Wilson CL, Stanford IM (2008) Functional characterization of GABAergic pallidopallidal and striatopallidal synapses in the rat globus pallidus in vitro. Eur J Neurosci 28:2401-2408. CrossRef Medline

Smith Y, Parent A (1988) Neurons of the subthalamic nucleus in primates display glutamate but not GABA immunoreactivity. Brain Res 453:353-356. CrossRef Medline

Stuart G (1999) Voltage-activated sodium channels amplify inhibition in neocortical pyramidal neurons. Nat Neurosci 2:144-150. CrossRef Medline

Tunstall MJ, Oorschot DE, Kean A, Wickens JR (2002) Inhibitory interactions between spiny projection neurons in the rat striatum. J Neurophysiol 88:1263-1269. Medline 